\title{
Complete transposition requires four active monomers in the Mu transposase tetramer
}

\author{
Tania A. Baker ${ }^{1}$, Elena Kremenstova, and Li Luo ${ }^{1}$ \\ Department of Biology and the ${ }^{1}$ Howard Hughes Medical Institute, Massachusetts Institute of Technology, Cambridge, \\ Massachusetts USA
}

\begin{abstract}
A tetramer of $\mathrm{Mu}$ transposase (MuA) cleaves and joins multiple DNA strands to promote transposition. Derivatives of $\mathrm{MuA}$ altered at two acidic residues that are conserved among transposases and retroviral integrases form tetramers but are defective in both cleavage and joining. These mutant proteins were used to analyze the contribution of individual monomers to the activity of the tetramer. The performance of different protein combinations demonstrates that not all monomers need to be catalytically competent for the complex to promote an individual cleavage or joining reaction. Furthermore, the results indicate that each pair of essential residues is probably donated to the active complex by a single monomer. Although stable, tetramers composed of a mixture of mutant and wild-type MuA generate products cleaved at only one end and with only one end joined to the target DNA. The abundance of these abortive products and the ratios of the two proteins in complexes stalled at different steps indicate that the complete reaction requires the activity of all four monomers. Thus, each subunit of MuA appears to use the conserved acidic amino acids to promote one DNA cleavage or one DNA joining reaction.
\end{abstract}

[Key Words: $\mathrm{Mu}$ transposase; transposition; protein multimers; genetic recombination]

Received July 7, 1994; revised version accepted August 18, 1994.

Transposition is the type of genetic recombination that moves a mobile DNA element from one site to another in the DNA of a host organism. The reaction proceeds through a series of DNA cleavage and joining reactions. Donor DNA cleavage separates the element from the host DNA at the old location while DNA strand transfer covalently joins the element DNA to the DNA at the new target site. Retroviruses and long terminal repeat (LTR) retrotransposons use a very similar mechanism to integrate a reverse-transcribed copy of their genomes into the DNA of the host cell. In most systems a single protein, the transposase or integrase, is responsible for both DNA cleavage and strand transfer. For phage $\mathrm{Mu}$, the transposase is the MuA protein (MuA).

The chemistry of transposition has been examined in some detail (for review, see Mizuuchi 1992a,b). Donor DNA cleavage uncovers a $3^{\prime}-\mathrm{OH}$ at each end of the transposon DNA. In some elements, including $\mathrm{Mu}$ and retroviruses, only one strand is cleaved /Craigie and Mizuuchi 1987; Surette et al. 1987; Fujiwara and Mizuuchi 1988; Brown et al. 1989; Craigie et al. 1990), whereas in others, such as $\operatorname{Tn} 10, \operatorname{Tn} 7$, and $P$ elements, donor cleavage involves double-stranded breaks (Bainton et al. 1991; Benjamin and Kleckner 1992; Kaufman and Rio 1992). In the subsequent strand transfer step, the two $3^{\prime}-\mathrm{OH}$ ends of the donor DNA are covalently joined to two $5^{\prime}$ phosphates, staggered by a few base pairs, in the two DNA strands at the target site. Strand transfer by the Mu transposase (Mizuuchi and Adzuma 1991) and the human immunodeficiency virus (HIV) integrase (Engelman et al. 1991) results in inversion of a chiral phosphorothioate at the site of insertion in the target DNA. Similarly, during donor cleavage by the HIV integrase, the chirality of a phosphorothioate at the cleavage site is inverted (Engelman et al. 1991). These results support the view that both cleavage and strand transfer proceed by a one-step phosphoryl transfer mechanism and are consistent with models in which the two reactions are catalyzed by a common active site (Engelman et al. 1991; Mizuuchi 1992b).

The similarities in the mechanism of transposition used by many elements extends to resemblance in the proteins that catalyze the reactions. Retroviral integrases have three highly conserved acidic amino acids termed the D-D-35-E motif, as the second aspartate (D) and the glutamate $(E)$ are separated by 35 residues; these residues of the HIV and Rous sarcoma virus (RSV) integrases are important for both cleavage and strand transfer in vitro (Drelich et al. 1992; Engelman and Craigie 1992; Kulkosky et al. 1992; Lefemina et al. 1992; van Gent et al. 1992; Leavitt et al. 1993). We have recently identified acidic residues in $\mathrm{MuA}$ that are required for both DNA cleavage and strand transfer (Baker and Luo 1994). The similar effect of mutations, as well as amino acid se- 
quence similarity, argue that the essential acidic amino acids of MuA (D269 and E392) are analogs of those of the retroviral integrases (Baker and Luo 1994). Convincing D-D-35-E motifs have been detected in many transposition proteins, including those from Tn7, Tn552, IS3, and mariner elements (Fayet et al. 1990; Rowland and Dyke 1990; Robertson 1993; Baker and Luo 1994; Rådström et al. 1994) and a related motif has been identified in the proteins encoded by excising elements from ciliated protozoa (Doak et al. 1994). Thus, MuA appears to be a member of a large protein family that utilizes a similar constellation of residues to promote genetic recombination. The essential acidic amino acids have been suggested to form part of the active site for both cleavage and strand transfer by coordinating divalent cations $\left(\mathrm{Mg}^{2+}\right.$ or $\mathrm{Mn}^{2+}$ ) (Engelman and Craigie 1992; Kulkosky et al. 1992; Bushman et al. 1993). Some activity is restored to the D269N and E392Q MuA derivatives by addition of high concentrations of $\mathrm{Mn}^{2+}$ in support of this suggestion (Baker and Luo 1994).

Successful transposition critically depends on coordination of donor cleavage and strand transfer on the two ends of the element. This coordination appears to be mediated by a multimeric complex of the transposase. Transposition of $\mathrm{Mu}, \mathrm{Tn} 10$, and $\mathrm{Tn} 7$ all involve higherorder complexes between the transposases and the donor and target DNAs (Surette et al. 1987; Haniford et al. 1991; Mizuuchi et al. 1992; Bainton et al. 1993). Retroviral integration also involves a multimeric complex of the integrase protein (Jones et al. 1992; Engelman et al. 1993; van Gent et al. 1993b). Characterization of these complexes is most advanced for $\mathrm{Mu}$, where it has been demonstrated that the transposition complex consists of a tetramer of $75-\mathrm{kD}$ MuA monomers bound simultaneously to the two ends of the Mu DNA (Lavoie et al. 1991; Mizuuchi et al. 1992). This tetramer pairs the two DNA ends and engages the DNA cleavage sites (Lavoie et al. 1991; Mizuuchi et al. 1991, 1992; Baker et al. 1993). Formation of the complex involves several MuA-binding sites on a supercoiled donor DNA and the host DNAbinding proteins $\mathrm{HU}$ and IHF (Surette and Chaconas 1989, 1992; Baker and Mizuuchi 1992; Mizuuchi, et al. 1992). Assembly appears to activate the catalytic activity of MuA, and once formed, the constituent monomers do not exchange with those in solution (Lavoie et al. 1991; Surette et al. 1991; Mizuuchi et al. 1992). Three stable MuA-DNA complexes have been characterized: The stable synaptic complex (SSC, or type 0 transpososome), which assembles on the donor DNA prior to cleavage, the cleaved donor complex $(\mathrm{CDC}$, or type 1 transpososome), and the strand transfer complex (STC, or type 2 transpososome). Transposition involves a second phageencoded protein, MuB. MuB interacts with the MuAdonor DNA complexes and stimulates cleavage and strand transfer by MuA (Baker et al. 1991; Surette and Chaconas 1991; Surette et al. 1991).

The knowledge that $\mathrm{Mu}$ transposition is promoted by a stable tetramer of MuA allows for a level of analysis of the transposition mechanism not yet possible in other systems. In this report we address how the four sets of essential acidic amino acids of the MuA tetramer contribute to the activity of the complex. The activities of the mixed tetramers containing wild-type $\mathrm{MuA}$ and mutant proteins altered at the essential acidic amino acids indicate that normal transposition requires catalytic activity of all four monomers. Thus, these data support models in which the acidic amino acids participate in both cleavage and strand transfer but different monomers carry out the two chemical steps.

\section{Results}

Mutant and wild-type MuA form mixed complexes that have partial activity.

To start to address how the four sets of essential acidic amino acids in the MuA tetramer contribute to the cleavage and strand transfer activity of the complex, the impact of the D269N and E392Q proteins on reactions containing wild-type MuA (WTMuA) was investigated. These proteins, altered at the conserved acidic amino acids, assemble with the donor DNA into stable complexes but are severely defective in both cleavage and strand transfer (Baker and Luo 1994). Two types of donor DNA were used to assay the protein mixtures: a circular plasmid containing two intact Mu DNA ends, and precleaved linear DNA, in which the sites of donor DNA cleavage have already been uncovered by digestion with HindIII. With the circular substrate, MuA nicks the DNA at two specific sites and then joins these $3^{\prime}-\mathrm{OH}$ ends to the target DNA by strand transfer. With the precleaved DNA, MuA carries out only strand transfer.

Cleavage of the circular substrate, and strand transfer of both types of donor DNA, were steeply dependent on the concentration of WTMuA; little product was observed with $<0.5$ pmole of MuA, whereas full activity was seen with 1.3 pmole. In reactions containing WTMuA with circular DNA, addition of E392Q (1.3 pmole) had two principal effects (Fig. 1): (1) Detectable donor cleavage occurred at WTMuA concentrations too low to support the reaction on their own; and (2) at higher levels of WTMuA, E392Q inhibited strand transfer, and the cleaved donor DNA accumulated. Addition of E392Q also affected strand transfer of precleaved donor DNA. E392Q stimulated strand transfer in the presence of low levels of WTMuA. Furthermore, the mutant protein changed the distribution of the strand transfer products; a single band with a migration distinct from the products formed by WTMuA was the principal product when both proteins were present. The following experiment addresses the possibility that this product resulted from strand transfer of only one of the two Mu DNA ends into the target DNA.

To probe the structure of the strand transfer products formed by the mixtures of WTMuA and an inactive mutant protein (here, D269N, E392Q, and D269N have qualitatively similar affects), restriction fragments carrying only the right end of the Mu DNA were used as the donor DNA. With this type of substrate, MuA efficiently pairs two fragments, and inserts them into a target site 


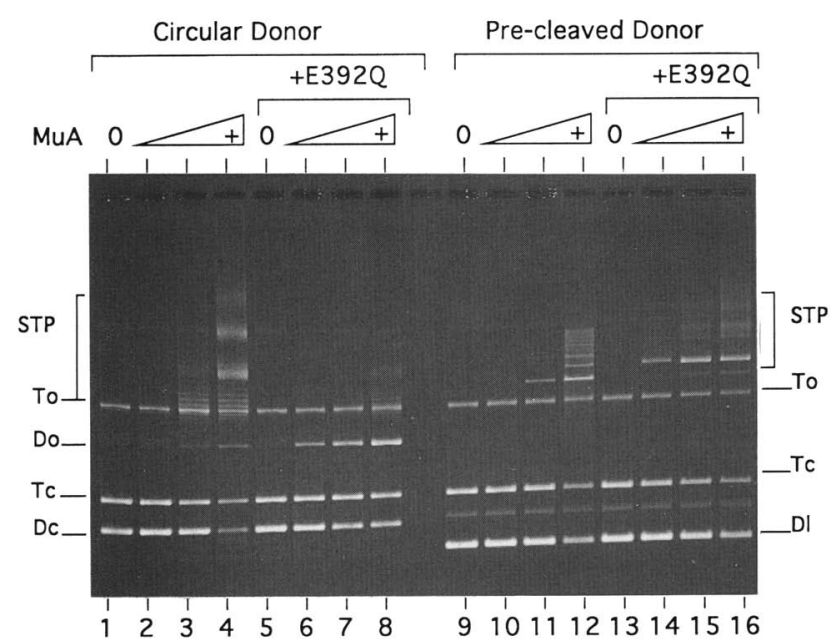

Figure 1. The activity of mixtures of MuA and $\mathrm{E} 392 \mathrm{Q}$ protein on supercoiled and precleaved DNAs. The different DNA species are labeled on the side of the gel. (Dc) The closed circular donor DNA; (Do) nicked donor DNA; (Dl) the linear (precleaved) donor DNA; (Tc and Tol The closed and nicked circular forms of the target DNA; (STP) The strand transfer products. Intermolecular strand transfer results in the slow migrating products, whereas intramolecular strand transfer results in the fast migrating species at the bottom of the gel. Reactions contained wild-type MuA alone or in the presence of 1.3 pmole of purified E392Q. The donor DNA was either supercoiled or precleaved as marked. Wild-type MuA levels were no WTMuA (lanes $1,5,9,13) ; 0.13$ pmole, (lanes $2,6,10,14) ; 0.46$ pmole, (lanes $3,7,11,15)$; and 1.3 pmoles, (lanes $4,8,12,16$ ).

(Craigie and Mizuuchi 1987; Mizuuchi and Adzuma 1991). Insertion of a pair of $\mathrm{Mu}$ end fragments into a circular target DNA (such as the supercoiled circular $\phi X 174$ DNA) generates a gapped linear molecule longer than the target DNA by the length of the two Mu end fragments (Fig. 2A). If only one Mu DNA end is joined, the product is a nicked circle with a covalently attached $\mathrm{Mu}$ end tail (a $\sigma$ structure). These two types of products are distinguished easily by agarose gel electrophoresis (Mizuuchi and Adzuma 1991).

Pre-cleaved $\mathrm{Mu}$ end fragments of two different lengths were used as the donor DNA in reactions containing different levels of WTMuA and D269N. When only WTMuA was present, three linear strand transfer products were generated as a result of the pair-wise joining of the three end fragment combinations (short + short, short + long, and long + long) (Fig. 2B). In contrast, when D269N (1.1 pmole) was present in addition to WTMuA the $\sigma$ products were observed. At low concentrations of WTMuA $(0.13$ pmole), these structures were the predominant product, whereas at the higher concentrations of WTMuA, the linear products were also evident. Thus, the efficiency of single-ended versus double-ended strand transfer of precleaved donor DNA depends on the ratio of WTMuA/D269N in the reaction.

The efficiency of cleavage at the two ends of the Mu sequences on a circular donor DNA by different ratios of mutant and WTMuA was also measured. When WTMuA is used alone, most of the nicked DNA is cleaved at both the left and right ends (Surette et al. 1991; Mizuuchi et al. 1992). In contrast, when $E 392 \mathrm{Q}$ or $\mathrm{D} 269 \mathrm{~N}$ is present in addition to WTMuA, a substantial amount of the nicked donor DNA remains uncleaved at one of the $\mathrm{Mu}$ DNA ends (data not shown). For example, in the presence of 0.13 or 0.43 pmole of WTMuA and 1.1 pmole of
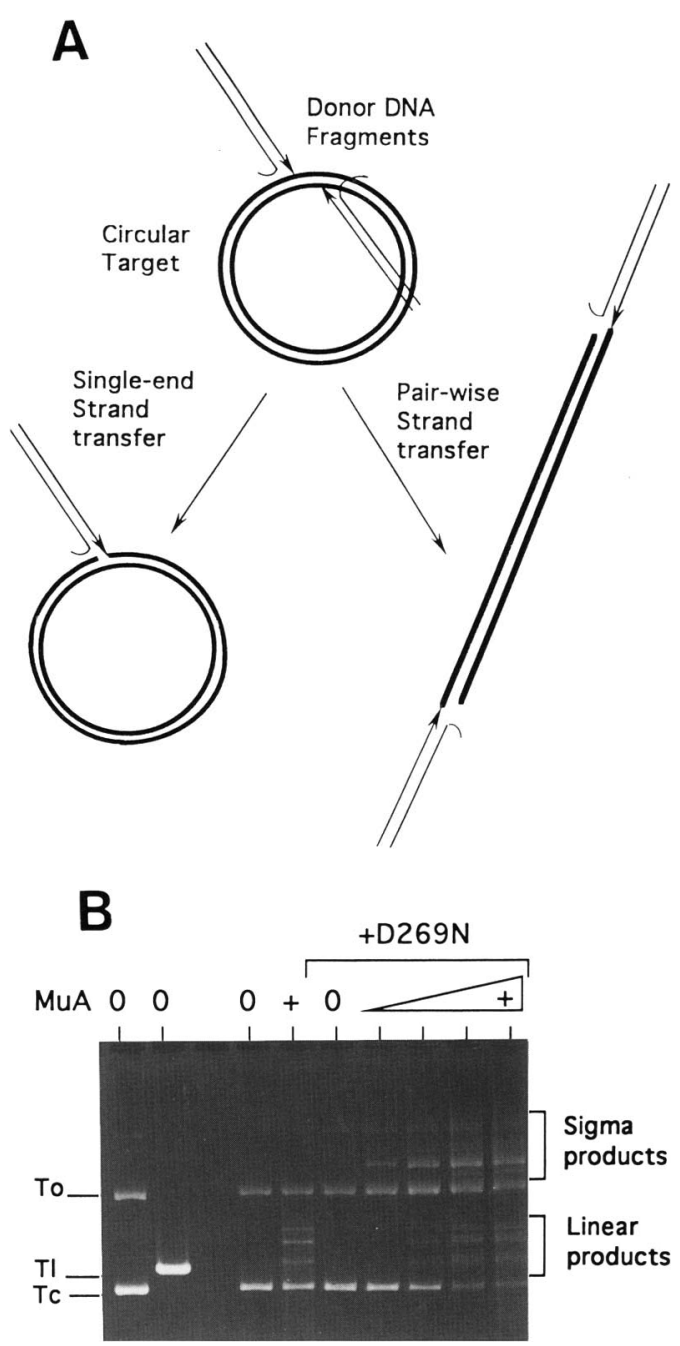

Figure 2. The efficiency of one-ended vs. two-ended strand transfer depends on the relative levels of MuA and D269N. $|A|$ Diagram of the products of a strand transfer reaction with linear Mu DNA end fragments as the donor DNA and a circular DNA as the target. The thin lines are the donor DNA, with the arrowheads showing the $3^{\prime}$ ends. The thicker lines illustrate the target DNA. $(B)$ Agarose gel of the strand transfer products made in reactions with different levels of MuA and D269N. Lanes 1 and 2 are markers showing the electrophoretic mobility of the nicked circular (To) and linear (Tl) forms of the target DNA. The donor DNA fragments are small and migrated off the gel. Protein levels in the reactions were as follows: (Lane 3) No MuA; (lane 4) 1.3 pmole of wild-type MuA; (lanes 5-9) 1.1 pmole of D269, in addition to the following levels of wild-type MuA: (lane 5) 0 pmole; (lane 6) 0.13 pmole; (lane 7) 0.46 pmole; (lane 8) 1.3 pmole; (lane 9) 2.6 pmoles. 
D269N, about half of the nicked DNA was cut at the left end and half was cut at the right end (values between $47 \%$ and $53 \%$. Thus, under these conditions, most of the cleaved donor DNA appears nicked at only one of the two $\mathrm{Mu}$ DNA ends, indicating that the mutant protein impedes cleavage when incorporated at certain positions in the tetramer.

The contribution of the acidic amino acids of MuA to the activities of the tetramer was investigated further by the assaying of both the double mutant protein and mixtures of the two mutant proteins. MuA mutated simultaneously at both essential amino acids /the D269N/ E392Q double mutant) had an impact on cleavage and strand transfer very similar to the single substitution proteins when mixed with WTMuA (Fig. 3). Therefore, in the context of the tetramer, monomers with either one or two altered acidic amino acids behave similarly. In contrast, mixing the $\mathrm{D} 269 \mathrm{~N}$ and $\mathrm{E} 392 \mathrm{Q}$ proteins did not yield any detectable activity with either the circular or precleaved donor substrate (data not shown). Taken together, these results indicate that D269 and E392 are probably donated to the active complex together by a single monomer, rather than D269 and E392 from different monomers working together in the final complex. How many monomers need the essential acidic
amino acids for the complete reaction?

\section{Possible models}

The activities of mixtures of different forms of MuA indicate that proteins lacking an essential acidic amino acid form mixed tetramers with WTMuA readily and that at least some of these complexes are able to promote

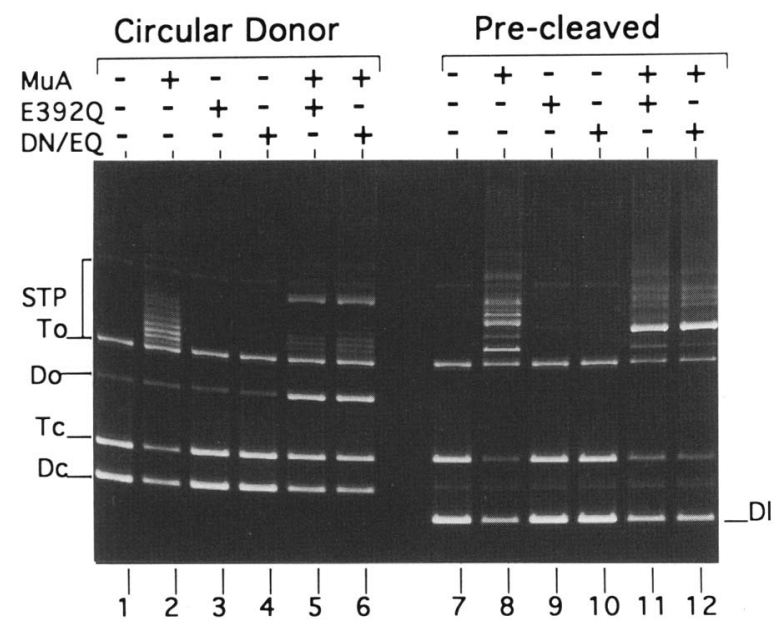

Figure 3. The D269N/E392Q double mutant protein is similar to single mutants in mixing experiments. The different DNA species are labeled on the side of the gel as described the legend to Fig. 1. Reactions contained wild-type MuA (1.3 pmole), E392Q (1.3 pmole), and/or D269N/E392Q (1.3 pmole) double mutant as shown at the top. (Lanes 1-6) Supercoiled donor DNA; (lanes 7-12) donor DNA was precleaved. an isolated cleavage or strand transfer event. However, these mixtures frequently generate aberrant products, indicating that incorporation of the mutant protein into the tetramer disrupts the normal sequence of chemical reactions. Therefore, we set out to address how many monomers in tetramer need D269 and E392 for the complex to form the normal strand transfer intermediate.

Formation of the strand transfer intermediate requires two DNA cleavages and two strand transfer reactions, one each on the left and right ends of the Mu DNA. In principle, one, two, three, or all four monomers might need to be active for the tetramer to catalyze these reactions. However, considering the symmetry of the reactions, and the fact that MuA is a tetramer, it seems most probable that either two or four catalytically active monomers are required. Furthermore, the characterization of the incomplete reaction products presented above argues against models involving one or three active monomers. If only one WTMuA monomer were necessary for the complete reaction, no accumulation of incomplete products by the mixed tetramers would be expected in contrast to the high frequency of aberrant products observed (unless the mixed complexes were unstable, which is tested below). Likewise, three subunit models stipulate that either one active monomer promotes cleavage of both ends of the Mu DNA /with the other two promoting strand transfer) or that one active monomer promotes strand transfer of both ends /with the other two required for cleavage). If one active monomer cleaves both ends, donor DNA cleaved at just one end would not be an expected product, in contrast to our findings. Similarly, we found that the mixed complexes promote single-end strand transfer of a precleaved DNA at high frequency, a reaction that would not be expected if one active monomer promoted strand transfer of both ends. Thus, different monomers appear responsible for the chemical reactions on the two Mu DNA ends, indicating that an even number of active subunits participate in the complete transposition reaction.

We therefore considered in detail models of the tetramer that involve two or four monomers participating in catalysis (Fig. 4). In Figure 4, the monomers are labeled $\mathrm{La}$ and $\mathrm{Lb}$ if they interact with the left end of the $\mathrm{Mu}$ $\mathrm{DNA}$ and $\mathrm{Ra}$ and $\mathrm{Rb}$ if they interact with the right end. In the four-subunit model, each monomer needs the essential acidic amino acids to perform one function, that is, cleavage or strand transfer of one end. The La monomer must be wild type for the complex to cleave the left end, whereas the Ra monomer must be wild type for cleavage of the right end; in addition, the $\mathrm{Lb}$ and $\mathrm{Rb}$ monomers also must be wild type for these cleaved ends to complete strand transfer. In contrast, in the two-subunit model the same monomer donates the acidic amino acids for both cleavage and strand transfer of one end of the $\mathrm{Mu} \mathrm{DNA}$ (the La and Ra monomers in Fig. 4; there is another type of two-active-monomer arrangement that will be discussed further below).

There are 16 possible configurations of two types of monomers in the tetramer (Fig. 4, bottom). (Shaded circles represent mutant $\mathrm{MuA}$, and unshaded circles repre- 
Figure 4. Model with all the types of mixed tetramers arranged in the two- and four-subunit models. Two possible arrangements of the core domains of MuA and the substrate DNAs are shown at the top. In the four-subunit model, the four copies of domain II in the MuA tetramer are shown with each subunit interacting with one of the four phosphodiester bonds in the donor and target DNAs that participate in the chemical steps $\left({ }^{*}\right)$. The $3^{\prime}-\mathrm{OH}$ ends generated by the $\mathrm{La}$ and $\mathrm{Ra}$ subunits during cleavage could attack the phosphodiesters activated by $\mathrm{Lb}$ and $\mathrm{Rb}$ during strand transfer; therefore all four monomers would need D269 and E392 for the complete strand transfer intermediate to be generated. In the two-subunit model, both the donor and target DNA are activated by the D269 and E392 residues in the La and Ra monomers; thus, $\mathrm{Rb}$ and $\mathrm{Lb}$ are not required to be wild-type monomers for the formation of the complete strand transfer intermediate. (Bottom) The mutant monomers are represented by the shadded in circles and WTMuA is shown by unshaded circles. The 16 arrangements of the tetramer were organized for both models on the basis of the products expected after reaction with a circular donor DNA substrate.
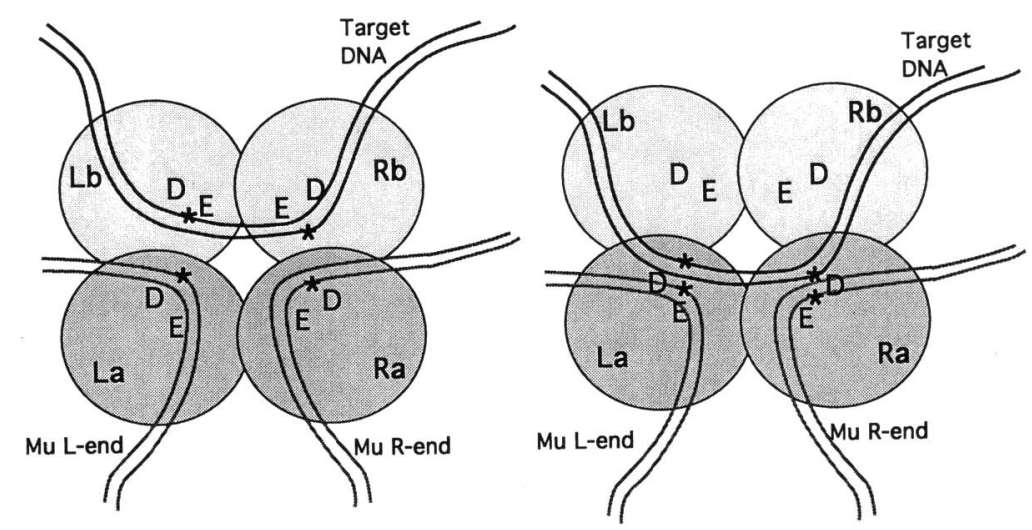

Four Subunit Model

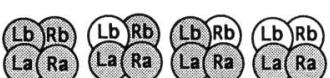
No Reaction Two Subunit Model (Lb) Rb) (Lb) Rb Rb (Lb)Rb La Ra La Ra La Ra la Ra

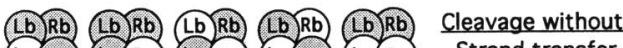
La Ra La Ra La Ra La Ra La Ra Strand transfer

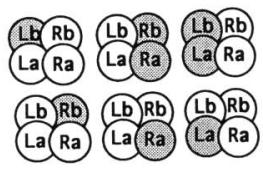

Single-end Strand transfer
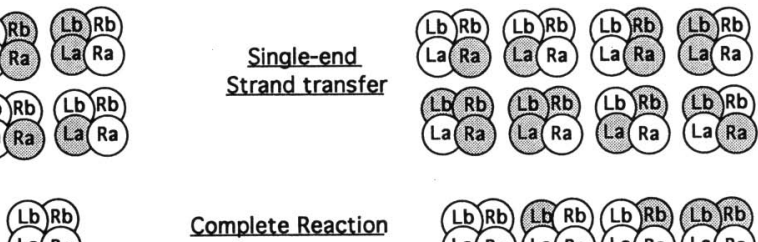
La Ra

Complete Reaction

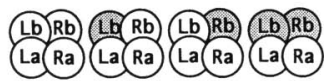

sent WTMuA.) These 16 tetramer arrangements were organized on the basis of the products they would be expected to generate after a complete reaction with a circular donor DNA using either the two- or four-subunit model (Fig. 4, bottom). A major difference between the two models is that the four-subunit arrangement predicts a class of complexes that are stalled after cleavage (assuming that cleavage is essentially irreversible), unable to carry out strand transfer for lack of a second active monomer in the correct position. However, in the two-subunit model, all of the complexes that cleave the DNA are predicted to complete strand transfer of their cleaved end. Inspection of the products formed by mixed complexes in Figures 1 and 3 reveals that in contrast to the situation with WTMuA alone and in apparent support of the four-subunit model, much of the donor DNA acted on by the mixed complexes appears stalled after cleavage. The reasons for this incomplete strand transfer are explored in the following section.

\section{Mixed tetramers are stable and some complexes slowly promote single-end strand transfer}

One possible explanation for inefficient strand transfer by the mixed tetramers is that cleaved donor complexes containing the mutant protein fall apart before completing strand transfer. Two potential sources of instability are the presence of the mutant protein and that only one of the Mu DNA ends is cleaved in most circumstances.
To directly observe transposition complexes containing $\mathrm{E} 392 \mathrm{Q}$, the protein was radiolabeled with $\left[{ }^{35}\right.$ S $]$ cysteine and $\left[{ }^{35} \mathrm{~S}\right]$ methionine. Reactions utilizing the purified labeled protein mixed with unlabeled WTMuA were subjected to electrophoresis on agarose gels both after addition of SDS to observe the DNA and without SDS to observe the MuA-DNA complexes (Fig. 5A). Nearly all of the cleaved DNA was found in the stable cleaved donor complex (CDC) rather than as free nicked DNA. Autoradiography revealed E392Q in this complex, as well as in the stable complex on the uncleaved donor DNA (SSC) and the strand transfer complex (STC). These data demonstrate that a majority of the cleaved complexes are stable for at least $1 \mathrm{hr}$. The complexes were also stable to heat denaturation, requiring incubation at temperatures higher than $60^{\circ} \mathrm{C}$ for $10 \mathrm{~min}$ to destroy them. They were, however, less thermostable than the CDC containing all WTMuA (data not shown).

Another possible explanation for incomplete strand transfer by the mixed tetramers is that strand transfer of one cleaved end might be slow or impossible if the other Mu DNA is uncleaved. Strand transfer by the mixed complexes was therefore compared with reactions containing WTMuA and a donor plasmid deleted for the cleavage site on the right end ( $\triangle \mathrm{RCS}$ plasmid) as another condition that may give rise to single-end strand transfer products (Fig. 5B). Although strand transfer with WTMuA on a normal donor DNA is usually complete within $10 \mathrm{~min}$, a predominant strand transfer product 
A

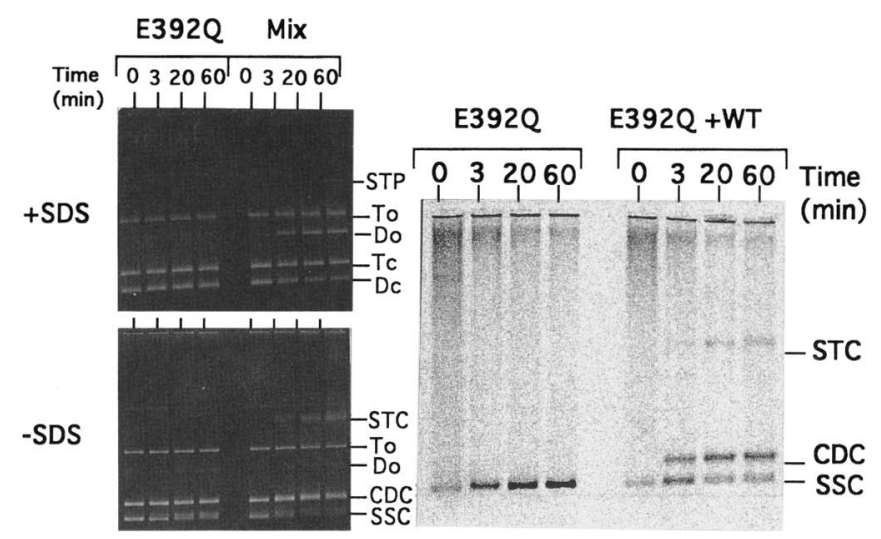

B

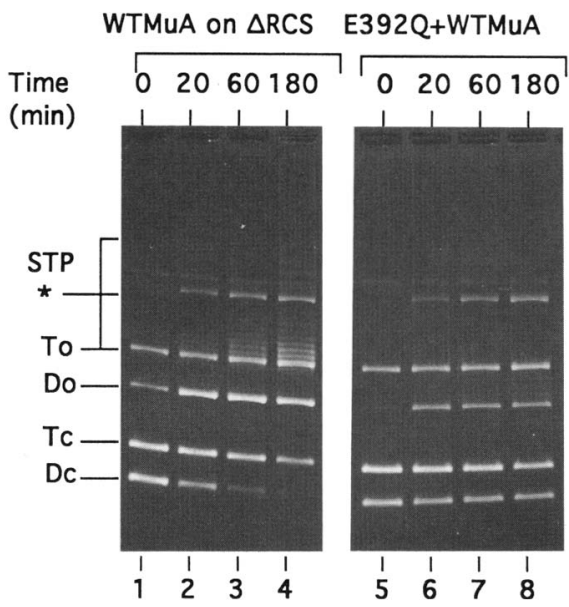

Figure 5. (A) Mixed tetramer complexes are stable. Reactions were done under standard conditions with either ${ }^{35} \mathrm{~S}-1$ abeled E392Q alone or a mixture of ${ }^{35} \mathrm{~S}$-labeled E392Q and WTMuA. Samples were removed for electrophoresis at the times shown at the top and run on agarose gels either with or without addition of SDS to the samples. Competitor DNA was added prior to electrophoresis as described in Materials and methods. The protein-DNA complexes are labeled as follows: (SSC) The stable synaptic complex, the MuA complex on the uncleaved donor DNA; (CDC) the cleaved donor complex; (STC) the strand transfer complex. The SSC migrates just slower than the supercoiled donor DNA (Do), whereas the CDC migrates in a manner similar to the supercoiled target DNA (To). The level of WTMuA present was 0.46 pmole; the concentration of the labeled E392Q protein was not determined carefully (for quantitative experiments, see Fig. 6). (B) Time course of single-end strand transfer. Reactions were done under standard reaction conditions and contained either a mixture of WTMuA (0.46 pmole) and E392Q (1.3 pmole) and the donor plasmid pSG1 or WTMuA (1.3 pmole) and the donor plasmid $\triangle R C S$. The different DNA species are labeled at left, as described in the legend to Fig. 1 and the single-end strand transfer product is labeled with an asterisk $(*)$. Changing the nucleotide sequence at one cleavage site does not completely block cleavage and strand transfer of this end (Surette et al. 1991); therefore, appearance of the normal double-end strand transfer products with the $\triangle \mathrm{RCS}$ plasmid was not surprising.

(marked with an asterisk in Fig. 5B) accumulated between 20 and $180 \mathrm{~min}$ in both experimental reactions. This product is not generally seen with WTMuA on a normal donor DNA (e.g., see Fig. 3A, lane 2). The mobility of the product suggested it may be the single-end strand transfer product. Double-end strand transfer products migrate as a family of bands because different numbers of superhelical turns in the donor DNA are trapped during strand transfer; covalent joining of only one end fails to trap these supercoils and therefore would yield a single product band.

The structure of the new strand transfer product $\left({ }^{*}\right)$ made by the E392Q/WTMuA mixtures was probed by digestion with restriction enzymes and two-dimensional agarose gel electrophoresis (data not shown). This analysis demonstrated that the DNA species consisted of the donor plasmid joined to the target DNA at only one end and that the left and right ends were joined at similar frequencies. In most of this class of products made by E392Q/WTMuA mixtures, the unjoined end was not cleaved (M. Mizuuchi, pers. comm.). The kinetics of reactions promoted by the E392Q/WTMuA mixture was also measured after addition of competitor DNA at 20 min. The rate and extent of strand transfer were unaffected by the competitor DNA, indicating that this single-end strand transfer product resulted from activity of stable complexes assembled within the first $20 \mathrm{~min}$ /data not shown). Accumulation of the single-end strand transfer product was essentially complete within $2 \mathrm{hr}$.
Thus, neither the instability of the WTMuA/E392Qcleaved complexes nor an indirect barrier to strand transfer of a cleaved end when the other end is uncleaved explain why the mixed tetramers fail to complete the normal sequence of cleavage and strand transfer reactions. In the following sections, the abundance of the different DNA products and the ratios of the mutant and wild-type proteins in complexes stalled at different steps are quantitated and compared with those predicted by the models.

The pattern of DNA products supports the four-subunit model

Four fates of the donor DNA can be directly quantitated from agarose gels: the unreacted DNA; the cleaved donor DNA; the single-end strand transfer product; and the double-end strand transfer products. The probability of forming each of these four products was calculated for ratios of E392Q/WTMuA of 9:1, 3:1, and 1:1 by use of the two- and four-subunit models outlined in Figure 4, and the predicted values were compared with those determined from quantitation of the reaction products observed on agarose gels (Table 1). The assumptions in the calculations were that (1) MuA is not limiting in the reactions, and (2) the tetramers form without bias to the position of the mutant and wild-type monomers. The observations that the left and right ends are left uncleaved by the mixed tetramers with similar frequency, 
Table 1. Fate of donor DNA in reactions containing different ratios of WTMuA and E392Q compared with predictions

\begin{tabular}{|c|c|c|c|c|}
\hline Ratio & Unreacted & Cleaved & Single-end transfer & Double-end transfer \\
\hline \multicolumn{5}{|l|}{$9: 1$} \\
\hline predicted 2 subunits & 81.0 & 0.0 & 18.0 & 1.0 \\
\hline predicted case $3-1$ & 90.0 & 8.1 & 1.8 & 0.11 \\
\hline predicted case $3-2$ & 81.0 & 15.4 & 2.7 & 0.21 \\
\hline predicted case $3-3$ & 81.0 & 15.4 & 2.5 & 0.41 \\
\hline predicted 4 subunits & 81.0 & 17.0 & 2.0 & 0.01 \\
\hline observed & $63.2 \pm 6.7$ & $18.1 \pm 7.8$ & $6.5 \pm 2.6$ & N.D. \\
\hline \multicolumn{5}{|l|}{$3: 1$} \\
\hline predicted 2 subunits & 56.1 & 0.0 & 37.4 & 6.3 \\
\hline predicted case $3-1$ & 74.8 & 14.0 & 9.4 & 1.6 \\
\hline predicted case $3-2$ & 56.1 & 24.5 & 14.1 & 2.8 \\
\hline predicted case $3-3$ & 56.1 & 24.5 & 11.7 & 5.2 \\
\hline predicted 4 subunits & 56.1 & 31.5 & 11.8 & 0.4 \\
\hline observed & $48.2 \pm 4.6$ & $35.7 \pm 8.9$ & $11.4 \pm 5.0$ & N.D. \\
\hline \multicolumn{5}{|l|}{$1: 1$} \\
\hline predicted 2 subunits & 25.0 & 0.0 & 50.0 & 25.0 \\
\hline predicted case $3-1$ & 50.0 & 12.5 & 25.0 & 12.5 \\
\hline predicted case $3-2$ & 25.0 & 18.8 & 37.5 & 18.8 \\
\hline predicted case $3-3$ & 25.0 & 18.8 & 25.0 & 31.2 \\
\hline predicted 4 subunits & 25.0 & 31.3 & 37.5 & 6.2 \\
\hline observed & $36.2 \pm 1.0$ & $34.8 \pm 10.4$ & $17.9 \pm 4.6$ & $2.8 \pm 4.9$ \\
\hline
\end{tabular}

The predicted values calculated for the four- and two-subunit models are for the subunit arrangements shown in Fig. 4 . The threesubunit arrangements are as follows: Case 3-1: La (or Ra) must be wild type for cleavage of both ends, whereas the activity of Ra (or La) has no effect; Lb must be wild type for strand transfer of the left end, and $\mathrm{Rb}$ must be wild type for strand transfer of the right end. Case 3-2: Either La or Ra must be wild type for both ends to be cleaved; Lb must be wild type for strand transfer of the left end and $\mathrm{Rb}$ must be wild type for strand transfer of the right end. Case 3-3: La must be wild type for cleavage of the left end. Ra must be wild type for cleavage of the right end; $\mathrm{Lb}$ or $\mathrm{Rb}$ (but only one of them) must be wild type for strand transfer of both ends. The percent of the donor DNA in each of the forms was quantitated from photographs of agarose gels as described in Materials and methods. Observed values are averages of between three and seven independent trials. The cleaved donor DNA present before the start of the reaction has been subtracted (usually between 5 and $13 \%$ of total). (N.D.) Not detectable by densitometry.

and that the two ends participate in single-end strand transfer approximately equally (data not shown), indicate that E392Q does not exhibit a strong assembly bias. Thus, random assembly of E392Q within the tetramer appears to be a reasonable approximation.

The observed distributions of DNA products supported the four-subunit model. At all three protein ratios a substantial amount of the donor DNA was cleaved rather than in the strand transfer product; the observed cleaved DNA agreed with the predictions of the foursubunit model within the error of the measurements (average error was $\pm 6 \%$ ). These data clearly favor the foursubunit model over the two-subunit model, which predicts complete conversion of the cleaved donor DNA to strand transfer products (see Fig. 4 and Table 1). Furthermore, a larger fraction of the donor DNA was converted to the strand transfer product when more WTMuA was present. Even if one disregards the unreacted DNA and considers only the efficiency of conversion of the cleaved DNA to the strand transfer products, more of the cleaved DNA was converted at the $1: 1$ ratio than in the $3: 1$ and $9: 1$ cases $(37.5 \%, 24.2 \%$, and $25.3 \%$ respectively). These data indicate that more WTMuA monomers are needed for cleavage followed by strand transfer than for cleavage alone, again in agreement with the four-subunit model.
The distribution of DNA products was also compared with the predictions of models in which three active subunits are required for transposition. Three specific three-subunit models are outlined in the footnote to $\mathrm{Ta}$ ble 1 . These models all predict substantially less cleaved DNA and more double-end strand transfer product than observed (see especially the 1:1 ratio). Thus, in addition to finding that the mixed tetramers promote both singleend cleavage and single-end strand transfer of a precleaved DNA, which argues against the three-subunit models (see above), this quantitative analysis also favors the four-subunit over the three-subunit arrangement.

Finally, an additional type of two-subunit model should be mentioned. An example of this type of model is that the Ra monomer could both cleave of the right end and promote strand transfer of the left end, whereas the La monomer cleaves the left end and promotes strand transfer of the right end. This type of model is also disqualified by the observed product distribution; in this case, the only abortive products predicted to accumulate are the two single-end cleavage products. If an active monomer were available to promote strand transfer of one cleaved end, it could also cleave the other end and pair-wise cleavage and strand transfer would be expected to be completed. 
The contribution of mutant and wild-type monomers in the stalled transposition complexes also supports the four-subunit model

The distribution of mutant and WTMuA in the stable MuA-DNA complexes was determined at the three different E392Q/WTMuA ratios. Either ${ }^{35}$ S-labeled E392Q or ${ }^{35} \mathrm{~S}$-labeled WTMuA was used in parallel reactions with the other protein unlabeled. The reaction products were electrophoresed both after addition of SDS to observe the DNA (Fig. 6, top) and without SDS to observe the MuA-DNA complexes (Fig. 6, bottom). A 40-fold excess of a competitor DNA oligonucleotide containing MuA-binding sites was added prior to running the gel; therefore, the protein in the complex bands reflects stably incorporated MuA. The ratio of E392Q/WTMuA in the complexes formed on the unreacted DNA, the cleaved DNA, and the strand transfer products was quantitated for the experiment shown in Figure 6 (Table 2).

The results of calculating the ratios of mutant and wild-type protein in the complexes can be summarized as follows. First, as the input ratio of E392Q to wild-type

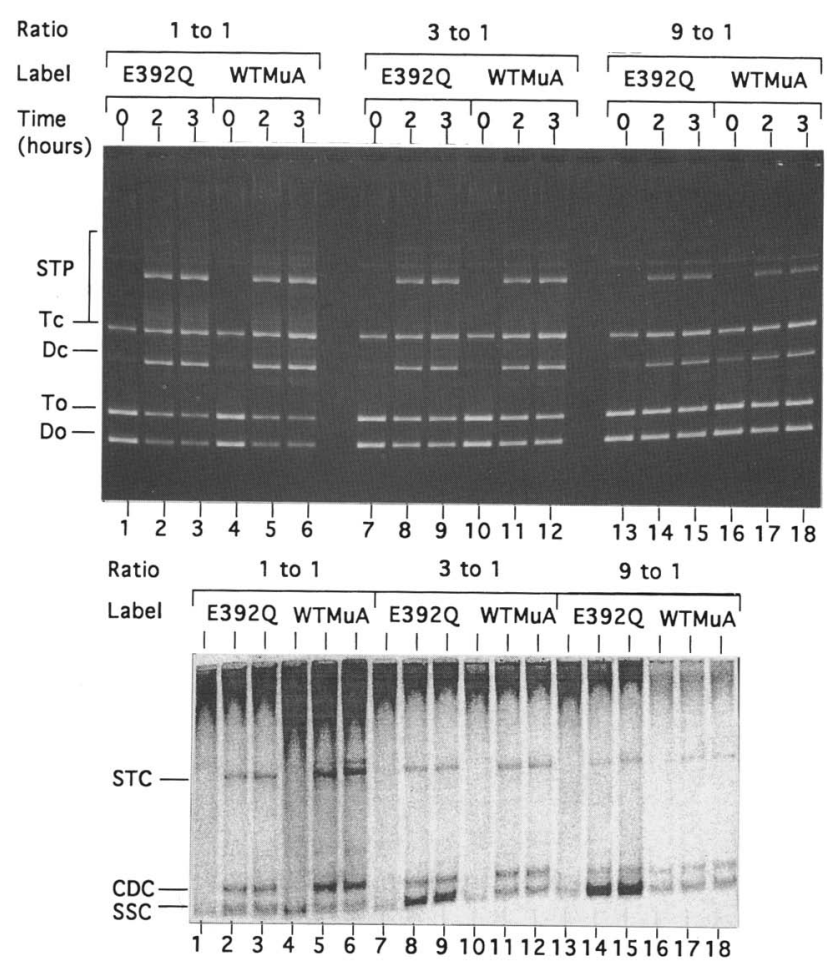

Figure 6. Composition of the transposition complexes formed at different ratios of E392Q and WTMuA. Reactions were done in parallel with either WTMuA or E392Q labeled with ${ }^{35} \mathrm{~S}$ and the other MuA derivative unlabeled as shown at the top. Three ratios of E392Q and WTMuA were investigated. All reactions contained 1.3 pmole of E392Q. At the $1: 1$ ratio, reactions also included 1.3 pmole of WTMuA, whereas the $3: 1$ ratio included 0.44 pmoles and the $9: 1$ ratio included 0.15 pmole of WTMuA. The specific radioactivity of WTMuA was 1.58 -fold higher than that of E392Q. In contrast to the DNA products, the single-end and double-end strand transfer complexes are not well separated during electrophoresis.
Table 2. Ratio of E392Q/WTMuA in the stable transposition complexes formed at different ratios of the two proteins

\begin{tabular}{lcccc}
\hline E392Q/WTMuA & $\begin{array}{c}\text { Time } \\
(\mathrm{hr})\end{array}$ & Unreacted & Cleaved & $\begin{array}{l}\text { Strand } \\
\text { transfer }\end{array}$ \\
\hline $1: 1$ & 2 & 2.2 & 1.0 & 0.66 \\
$1: 1$ & 3 & 2.2 & 0.68 & 0.68 \\
$3: 1$ & 2 & 4.7 & 1.5 & 1.2 \\
$3: 1$ & 3 & 4.9 & 1.6 & 1.1 \\
$9: 1$ & 2 & 9.3 & 2.8 & 1.4 \\
$9: 1$ & 3 & 9.6 & 2.8 & 1.1 \\
\hline
\end{tabular}

The relative amounts of the mutant and wild-type proteins in the different stable complexes were determined by exposure of the dried agarose gel shown in Fig. 6A to a PhosphorImager screen for several days and determination of the intensities in the various complex bands. The background was determined by quantitation of the intensity of the whole lane, and the emergence of the complex peaks above this background was used to calculate the values. This type of background measurement was necessary as the background varied between different lanes and within a lane, depending on how much labeled protein was present. These values were then corrected for the difference in specific radioactivity of the two protein preparations, and the value for the mutant protein was divided by that of the wildtype protein. The experiment was repeated several times with similar results.

protein was increased to nine, the E392Q/WTMuA ratio in the cleaved donor complexes approached three. These data indicate that tetramers consisting of one wild-type and three mutant monomers can cleave the donor DNA at one end. Second, at both the 3:1 and 9:1 ratios of E392Q/WTMuA, where almost all of the strand transfer product is joined at only one end (see above), nearly equal amounts of the two proteins were found in the strand transfer complexes. Thus, complexes that promote cleavage and single-end strand transfer appear to consist of two mutant and two wild-type monomers. A logical extension of these observations is that pair-wise cleavage and strand transfer of the two $\mathrm{Mu}$ DNA ends requires four active monomers of MuA.

\section{Discussion}

Complete transposition requires four active monomers of $M u A$

Catalytically compromised MuA derivatives altered at the acidic amino acids conserved among many transposases and retroviral integrases were used to probe the role of individual monomers in the transposition complex. Tetramers containing the mutant and wild-type proteins assemble and are active but frequently generate incomplete recombination products. Formation of the different classes of abortive products argues that four active monomers participate in generating the complete strand transfer intermediate. Several observations support this conclusion. (1) Pair-wise strand transfer of two $\mathrm{Mu}$ end fragments by mutant/wild-type protein mixtures requires more WTMuA than does single-end strand 
transfer, indicating that a different monomer promotes the reaction at each of the two $\mathrm{Mu}$ DNA ends. (2) The mixed tetramers often cleave a circular donor DNA at just one end indicating that different monomers cleave each end as well. (3) Some of the mixed tetramers carry out cleavage and single-end strand transfer, whereas others are stalled after cleavage. The fraction that completes strand transfer is higher in reactions containing more WTMuA, indicating that an additional active monomer is needed for cleavage and strand transfer than for cleavage alone. (4) When WTMuA is limiting, the mixed complexes stalled after cleavage have, on average, one wildtype and three mutant monomers, whereas those that complete single-end strand transfer appear to contain two mutant and two wild-type proteins. Thus, each subunit of the MuA tetramer appears to require the conserved acidic amino acids to promote one cleavage or one strand transfer reaction.

Analysis of the chemistry of donor cleavage and strand transfer indicates that the same active site in transposases and retroviral integrases could promote both reactions (Engelman et al. 1991; Vink et al. 1991; van Gent et al. 1993a). Furthermore, most mutations in the conserved acidic residues of HIV and RSV integrase, and MuA, affect both cleavage and strand transfer in parallel (Drelich et al. 1992; Engelman and Craigie 1992; Kulkosky et al. 1992; Lefemina et al. 1992; van Gent et al. 1992; Leavitt et al. 1993; Baker and Luo 1994). Thus, as has been suggested previously, the essential acidic amino acids may form part of the active site for both steps, with the major difference between the two reactions being whether water or the $3^{\prime}-\mathrm{OH}$ end of the donor DNA is chosen as the nucleophile that attacks the DNA phosphodiester bond (Engelman et al. 1991; Mizuuchi and Adzuma 1991; Mizuuchi 1992a). Therefore, our finding that four wild-type monomers are needed for the complete transposition reaction supports a model proposed previously for HIV integrase on the basis of analysis of the chemistry of the two steps, in which each monomer catalyzes the reaction at one of the four participating phosphodiester bonds (Engelman et al. 1991; Mizuuchi 1992a). However, our data do not address whether the essential acidic residues in the four monomers participate in the same capacity for all four reactions, only that the complete reaction is most efficiently promoted by the fully wild-type complex. It is also possible that these residues do not participate directly in catalysis of either cleavage or strand transfer, or that those from one dimer catalyzes both steps but the second dimer needs to have the wild-type residues to make a conformational change required for the complete reaction. Although these arrangements are possible, we do not consider them to be the most economical.

The behavior of MuA tetramers containing mixtures of D269N or E392Q and WTMuA complement and extend the results of a similar analysis with an inactive deletion protein (Baker et al. 1993). Like D269N and E392Q, MuA protein lacking the carboxy-terminal domain (MuAl-574) is defective in donor cleavage and strand transfer (Baker et al. 1993). The behavior of
MuA1-574 mixed with WTMuA on a supercoiled donor DNA is also similar to that of the point mutants: MuAl574 stimulates donor cleavage in the presence of low levels of WTMuA but inhibits strand transfer, resulting in the accumulation of the cleaved donor complex (Baker et al. 1993). Therefore, like the experiments presented here, the activity of mixtures of MuAl-574 and WTMuA indicate that two monomers are principally responsible for cleavage and two different monomers are responsible for strand transfer.

Distinctions in the behavior of MuAl-574 and D269N and E392Q also provide a hint about the possible roles of these regions. In contrast to the point mutations, $\mathrm{MuAl}$ 574 does not form tetramers in the presence of $\mathrm{Mu}$ end DNA. Mixed tetramers with MuAl-574 and WTMuA form, but MuAl-574 appears to avoid (during assembly) the positions in the tetramer that cleave the donor DNA. As a result, most of the MuA1-574/WTMuA-cleaved complexes are cleaved at both ends but unable to promote strand transfer. These results suggested that domain IIIA (which is missing from MuAl-574) is involved in contacting the cleavage sites on the donor DNA during tetramer assembly and the site of insertion on the target DNA during strand transfer (Baker et al. 1993). In contrast, the mutants altered at the essential acidic amino acids do not appear to be severely defective in contacting the cleavage site or the target DNA, as inferred from their ability to assemble and the activity of the mixed complexes. Thus, the D269N and E392Q proteins are probably defective in a function distinct from that defective in MuA1-574, and this function is needed at a later stage of the reaction.

In addition to implicating all four monomers in catalysis, the mixing experiments provide some additional clues about the organization of the active transposase complex. When mixed with WTMuA, the E392Q/ D269N double mutant has similar activity to the E392Q or $\mathrm{D} 269 \mathrm{~N}$ single mutant proteins. Furthermore, mixtures of the two single substitution mutant proteins do not have detectable activity. These results argue against arrangements in which D269 from one monomer acts together with E392 of another monomer in the active complex. In this type of arrangement, the double mutant protein would be expected to be more defective than the single mutants in mixtures with WTMuA (inactivating two catalytic centers), whereas the two single substitution proteins, together, might form a partially active complex. By similar logic, the mixing of proteins mutated at residues of the yeast $2 \mu$ plasmid's site-specific recombinase (FLP) essential for catalysis (the active site Tyr and an Arg, His, Arg triad) indicates that the tyrosine and the triad are donated from different monomers to form one active site (Chen et al. 1992, 1993). This type of fractional active site may be common to the Int family of site-specific recombinases (Han et al. 1993) and ensures that the protein is not catalytically active until the complex is assembled. MuA and HIV integrase, like FLP, may be activated by multimerization; however, assembly of the essential acidic amino acids from different monomers does not seem to be the basis of this activation. As 
with $\mathrm{MuA}$, mixing two mutant $\mathrm{HIV}$ integrase proteins carrying changes in two different residues of the D-D35-E triad does not generate an active complex (van Gent et al. 1993b). However, assembly of a catalytic center from different monomers is still an attractive possibility with the transposases and integrases. Certain pairs of inactive integrase proteins with alterations in different domains have nearly wild-type activity when mixed (Engelman et al. 1993; van Gent et al. 1993b). Furthermore, mixtures of MuA1-574 and E392Q have strand transfer activity, indicating that although both proteins are inactive, they appear to form catalytically competent mixed tetramers (T. Baker, unpubl.).

\section{Comparison of the $\mathrm{Mu}$ transposase to other recombi- nase complexes}

The emerging relationships between both the mechanism of transposition and the transposition proteins encoded by different elements make it tempting to speculate about the organization of other transposases based on the results with $\mathrm{MuA}$. Although the multimeric state of HIV integrase is not known, kinetic data suggest that the active form is a dimer (Jones et al. 1992) and both dimers and tetramers have been detected by protein-protein cross-linking (Engelman et al. 1993). In vitro, HIV integrase promotes almost exclusively single-end cleavage and strand transfer (Engelman et al. 1993; van Gent et al. 1993b), in contrast to WTMuA, which nearly always promotes cleavage and strand transfer of a pair of ends together (Craigie and Mizuuchi 1985). If an integrase dimer promotes the reactions on a single end, perhaps a tetramer promotes the two cleavages and twostrand transfer reactions necessary for viral integration with the essential acidic amino acids from each monomer participating. In contrast to $\mathrm{Mu}$ and $\mathrm{HIV}$, some transposable elements, such as $\operatorname{Tn} 10, \operatorname{Tn} 7$ and $\mathrm{P}$ elements, cleave both strands of the element-host DNA junction prior to strand transfer of the cleaved $3^{\prime}$ ends into the target DNA (Bainton et al. 1991; Benjamin and Kleckner 1992; Kaufman and Rio 1992). Transposition of these elements involves a total of six DNA phosphoryl transfer reactions, four cleavages, and two strand transfers. Thus, a more complex arrangement of the active multimer may be required. Perhaps the D-D-35-E motif proteins are flexible enough to assemble into a complex that can promote all of these steps. For example, Tn7 encodes two proteins with D-D-35-E motifs, TnsB and TnsA. Both proteins are required for donor cleavage and strand transfer, but altering the acidic amino acids in TnsA blocks one set of donor DNA cleavages, without effecting cleavage or strand transfer of the other strands (N. Craig, pers. comm.). Two alternative arrangements for the transposes subunits are attractive: (1) The active form may be a hexamer with one subunit promoting each reaction; or (2) the active form may be a tetramer, with one dimer promoting two sets of reactions.

Multimeric protein-DNA complexes promote many important biological reactions, including initiation of transcription, DNA replication, and genetic recombina- tion. Of these complexes, those that catalyze site-specific recombination and transposition are among the best characterized. These multimeric complexes bring together distantly located DNA segments to participate in the reaction. Assembly of the multimeric proteins is also a common site of control, preventing reactions from initiating if they will cause toxic genetic rearrangements or are destined to be incomplete (Gellert and Nash 1987; Mizuuchi et al. 1992). The individual protein components of multimeric complexes may function as architectural elements, adapter elements (that communicate between subunits), and catalytic components. The assaying of complexes containing altered subunits can uncover these roles. As described above, analysis of FLP mutant mixtures has illuminated the elegant strategy of assembling active sites from multiple subunits to couple construction of the complex to catalysis (Chen et al. 1992, 1993). In this case, although no monomer has a complete active site, each monomer contributes to the catalytic activity of the complex. Similar experiments with another site-specific recombinase, the $\gamma \delta$ resolvase, reveal that 4 subunits bound to a specific set of DNA sites within the 12-subunit multimer must be catalytically competent, whereas other monomers mediate synapsis of the recombination DNAs (Grindley 1993). In Mu transposition, the monomers in the stable tetramer both pair the recombining DNAs and catalyze the chemical steps of the reaction (Lavoie et al. 1991; Mizuuchi et al. 1991, 1992). This tetrameric core is also the target of activation by the MuB protein (Baker et al. 1991; Surette and Chaconas 1991), which directs transposition to distant target sites (Adzuma and Mizuuchi 1988), although which monomers interact with $\mathrm{MuB}$ is not yet clear. Clearly, our ability to construct protein-DNA complexes with altered subunits at different positions will continue to uncover the elegant ways in which biological catalysts are organized.

\section{Materials and methods}

\section{DNA}

The target DNA was $\phi \mathrm{Xl} 74 \mathrm{RFI}$ (BRL). The circular donor DNA was pSG1 for most experiments (Baker and Luo 1994). The precleaved donor DNA was made by linearization of pKN37 (identical to pMK426 between the two Mu DNA ends/ with HindIII as described previously (Craigie and Mizuuchi 1987). The donor DNA fragments used in Figure 2 were made by digestion of pKN37 with HindIII and BamHI. The $\triangle$ RCS plasmid is a derivative of pSGI, which carries the following modification. In pSG1, the sequence from the R1 MuA-binding site to the cleavage site is Rl. . .5'-CTTCA *TATGAATTC, whereas in $\triangle$ RCS the sequence is R1...5'-TAAGG*AATTC. The EcoRIsite used for cloning the synthetic fragments into pMK590 (which lacks the $\mathrm{Mu}$ right end but carries the left end) is shown in boldface type. The natural MuA cleavage site in pSG1 is shown by the asterisk, and the analogous position is marked in the $\triangle R C S$ sequence. $\triangle \mathrm{RCS}$ has one other difference from pSG1. The R3 MuA-binding site actually has the sequence of the L3 repeat. This change has little effect on the donor DNA activity of the plasmid in the absence of the change at the cleavage site ( $T$. Baker, unpubl.). 


\section{Proteins}

$\mathrm{MuB}$ protein was purified as described by Chaconas et al. (1985), with the additional step described by Adzuma and Mizuuchi (1991) to remove aggregated protein. HU protein was purified from the overproducing strain of R. McMacken (Johns Hopkins University, Baltimore, MD) by the method of Dixon and Kornberg (1984) with an additional step of Mono S chromatography in $20 \mathrm{~mm} \mathrm{NaPO}$ ( $\mathrm{pH} 4.8)$, eluted with a gradient of 0-500 mM $\mathrm{NaCl}$. MuA protein, D296N, E392Q, and D269N/E392Q were purified as described in Baker et al. (1993). The protein concentration of MuA preparations was determined spectrophotometrically with the value of $\epsilon 280=1.58$ for $1 \mathrm{mg} / \mathrm{ml}$. The concentration of the ${ }^{35} \mathrm{~S}$-labeled preparations was measured by use of the Coomassie brilliant blue-dye binding method (Bio-Rad) with purified WTMUA as a standard.

A plasmid expressing the double mutant (D269N/E392Q) was constructed by fragment swapping with the two single mutants (D269N and E392Q) cloned into the T7 RNA polymerase expression vector pMK591 (Baker and Luo 1994). Both plasmids that contain the single mutant were subjected to digestion by BamHI and BssHII. The 947-bp BssHII-BamHI fragment that carries E392Q was swapped into the vector that contains the D269N mutation. The presence of both mutations in the products was confirmed by sequencing with the Sequenase version 2 kit (U.S. Biochemical).

Both the wild-type MuA and the E392Q mutant were radiolabeled in vivo, and the labeled proteins were then purified according to the method described in Baker et al. (1993), except that the source of ${ }^{35} \mathrm{~S}$ used was the EXPRE ${ }^{35} \mathrm{~S}^{35} \mathrm{~S}$ Protein Labeling Mix, from Du Pont. To label $100 \mathrm{ml}$ of cell culture, $2 \mathrm{mCi}$ was used.

\section{Transposition reactions}

The standard reaction conditions were essentially as described previously (Craigie et al. 1985). Reaction mixtures contained 25 $\mathrm{mM}$ Tris- $\mathrm{Cl}$ (pH 8 at room temperature), $156 \mathrm{mM} \mathrm{NaCl}, 10 \mathrm{mM}$ $\mathrm{MgCl}_{2}, 2 \mathrm{mM}$ ATP, $1 \mathrm{~mm} \mathrm{DTT}$ /omitted in some reactions), $15 \%$ glycerol, $25 \mu \mathrm{g} / \mathrm{ml}$ of BSA, $10 \mu \mathrm{g} / \mathrm{ml}$ of donor DNA, and 10 $\mu \mathrm{g} / \mathrm{ml}$ of $\phi X 174$ RFI. The protein levels in $25-\mu 1$ reactions were as follows: MuB, 6.5 pmoles; hydroxyurea (HU), 3.0 pmoles; and $\mathrm{MuA}$, between 0.13 and 3.0 pmoles (see figure legends). Proteins were added by dilution of a stock solution into a buffer similar to the storage solution and addition of $1 \mu \mathrm{l}$. The dilution buffers were as described previously (Baker et al. 1991). The reactions were incubated at $30^{\circ} \mathrm{C}$ for $20 \mathrm{~min}$ for as specified in the legends). Reactions were stopped by the addition of 0.2 volume of a stop solution $(0.1 \%$ bromophenol blue, $2.5 \%$ SDS, $50 \mathrm{~mm}$ EDTA, $25 \%$ glyceroll, and portions of the samples were analyzed by electrophoresis on a $0.9 \%$ agarose gel with constant circulation of the buffer $(1 \times \mathrm{TAB}: 40 \mathrm{~mm}$ Tris acetate at $\mathrm{pH} 7.8$, $5 \mathrm{~mm}$ sodium acetate, $1 \mathrm{mM}$ EDTA).

For analysis of the stable MuA-DNA complexes by agarose gel electrophoresis, the reactions were done as described above except after incubation at $30^{\circ} \mathrm{C}$, the competitor DNA oligonucleotide (described previously in Mizuuchi et al. 1991) was added and the reactions were incubated for an additional $5 \mathrm{~min}$ at room temperature. The reactions were then stopped by addition of 0.2 volume of $80 \%$ glycerol and $50 \mathrm{~mm}$ EDTA, and electrophoresis was done under the same conditions as for the DNA products.

\section{Quantitation of DNA products and labeled proteins in complexes}

The percent of the total donor DNA in each of the types of DNA products was quantitated from a scanning negative of the ethiduim bromide-stained gels essentially as described previously (Mizuuchi et al. 1992) except an LKB 2202 Ultroscan Laser Densitometer with a Hewlett Packard 3390A Integrator was used to collect the data. Each lane of the negative was scanned at two or three positions, and the values were averaged.

The percent distribution of ${ }^{35} \mathrm{~S}$-labeled E392Q and WTMuA in the stable MuA donor DNA complexes was determined by drying the agarose gels and exposing them to a PhosphorImager screen for several days. Band intensities were measured by a Molecular Dynamics PhosphorImager. The relative specific radioactivity of the two protein preparations was determined by scintillation counting.

\section{Calculation of predicted DNA products}

The probability of forming each of the 16 types of tetramers shown in Figure 4 was calculated for the different input ratios of mutant to WTMuA of $1: 1,3: 1$, and 9:1. In each case the probability $(P)$ that a given monomer was mutant or wild type was determined from the input ratio of the two proteins. The following values were used: for the $1: 1$ ratio, $P_{\text {mut }}=0.5$ and $P_{\mathrm{wt}}=0.5$; for the $3: 1$ ratio, $P_{\mathrm{mut}}=0.75$ and $P_{\mathrm{wt}}=0.25$; and for the 9:1 ratio, $P_{\text {muc }}=0.9$ and $P_{\mathrm{wt}}=0.1$. The probability of forming each of the types of tetramers was then calculated. For example, the probability of forming the all wild-type tetramer is $\left(P_{\mathrm{wt}}\right)^{4}$, or $(0.25)^{4}=0.0039$ at the input protein ratio of $3: 1$, whereas the probability of a mixed tetramer containing two mutant and two wild-type monomers was $\left(P_{\text {wt }}\right)^{2}\left(P_{\text {mut }}\right)^{2}$ or $(0.25)^{2}(0.75)^{2}=0.035$. The tetramers were arranged in the two ways shown in Figure 4 on the basis of the type of product they would be expected to generate after a complete reaction with the circular donor DNA. The probabilities of forming each of the types of tetramers in the no-reaction, cleaved, single-end strand transfer, and complete reaction classes were calculated, and the values were summed and multiplied by 100 to give the percent of the total donor DNA expected to be in each class.

\section{Measuring the efficiency of cleavage of each $\mathrm{Mu} D \mathrm{DNA}$ end}

The efficiency of cleavage of the left and right ends of the $\mathrm{Mu}$ DNA by mixtures of MuA and D269N was measured as follows. Standard transposition reactions were prepared except that ATP, MuB, and target DNA were omitted. The reaction mixtures were stopped as described above, run on a $0.8 \%$ low-melting-temperature agarose gel, and the nicked DNA band was cut out of the gel. The DNA was recovered from the gel by use of $\beta$-agarase (New England Biolabs) and ethanol precipitation. Samples were then divided in half. To measure cleavage of the right end, the DNA was digested with AvaI, treated with phosphatase, and end-labeled with $\left[\gamma^{32} \mathrm{P}\right] \mathrm{ATP}$. After removal of the unincorporated ATP with G25 spin columns, the DNA was digested with EcoRI and run on a $6 \%$ denaturing polyacrylamide gel. Samples to measure cleavage of the left end were processed in parallel, except digestion was with ClaI followed by EcoNI. The uncut fragment on the right end was 58 nucleotides; cleavage by MuA shortened this to 50 nucleotides. The uncut left end fragment was 255 nucleotides, and the cut fragment was 201 nucleotides. The percent of the DNA cleaved at each end was determined by measuring the relative intensities of these bands with the Molecular Dynamics PhosphorImager.

\section{Acknowledgments}

We thank Alan Engelman, Kiyoshi and Michiyo Mizuuchi, Howard Nash, David Pincus, David Roth, and Tracy Smith for 
helpful comments on the manuscript. This work was supported by the Robert A. Swanson Career Development Professorship and U.S. Public Health Service grant GM49224 from The National Institutes of Health to T.A.B.

The publication costs of this article were defrayed in part by payment of page charges. This article must therefore be hereby marked advertisement" in accordance with 18 USC section 1734 solely to indicate this fact.

\section{References}

Adzuma, K. and K. Mizuuchi. 1988. Target immunity of $\mathrm{Mu}$ transposition reflects a differential distribution of $\mathrm{Mu} B$ protein. Cell 53: 257-266.

Bainton, R., P. Gamas, and N.L. Craig. 1991. Tn7 transposition in vitro proceeds through an excised transposon intermediate generated by staggered breaks in DNA. Cell 65: 805-816.

Bainton, R.J., K.M. Kubo, J. Feng, and N.L. Craig. 1993. Tn7 transposition: Target DNA recognition is mediated by multiple $\mathrm{Tn} 7$-encoded protein in a purified in vitro system. Cell 72: 931-943.

Baker, T.A. and L. Luo. 1994. Identification of residues in the Mu transposase essential for catalysis. Proc. Natl. Acad. Sci. 91: $6654-6658$.

Baker, T.A. and K. Mizuuchi. 1992. DNA-promoted assembly of the active tetramer of the $\mathrm{Mu}$ transposase. Genes \& Dev. 6: 2221-2232.

Baker, T.A., M. Mizuuchi, and K. Mizuuchi. 1991. MuB protein allosterically activates strand transfer by the transposase of phage Mu. Cell 65: 1003-1013.

Baker, T.A., M. Mizuuchi, H. Savilahti, and K. Mizuuchi. 1993. Division of labor among monomers within the $\mathrm{Mu}$ transposase tetramer. Cell 74: 723-733.

Benjamin, H.W. and N. Kleckner. 1992. Tn10 transposase excises Tn10 from flanking donor DNA by flush double strand cleavages at the transposon termini. Proc. Natl. Acad. Sci. 89: 4648-4652.

Brown, P.O., B. Bowerman, H.E. Varmus, and J.M. Bishop. 1989. Retroviral integration: Structure of the initial covalent product and its precursor, and a role for the viral IN protein. Proc. Natl. Acad. Sci. 86: 2525-2529.

Bushman, R.D., A. Engelman, I. Palmer, P. Wingfield, and R. Craigie. 1993. Domains of the integrase protein of human immunodeficiency virus type 1 responsible for polynucleotidyl transfer and zinc binding. Proc. Natl. Acad. Sci. 90: 3428-3432.

Chaconas, G., G. Gloor, and J.L. Miller. 1985. Amplification and purification of the bacteriophage $\mathrm{Mu}$ encoded $\mathrm{B}$ transposition protein. J. Biol. Chem. 260: 2662-2669.

Chen, J.W., J. Lee, and M. Jayaram. 1992. DNA cleavage in trans by the active site tyrosine during Flp recombination: Switching protein partners before exchanging strands. Cell 69: 647658.

Chen, J.W., S.H. Yang, and M. Jayaram. 1993. Tests for the fractional active-site model in Flp site-specific recombination. $J$. Biol. Chem. 268: 14417-14425.

Craigie, R. and K. Mizuuchi. 1985. Mechanism of transposition of bacteriophage $\mathrm{Mu}$ : Structure of a transposition intermediate. Cell 41: 867-876.

- 1987. Transposition of Mu DNA: Joining of Mu to target DNA can be uncoupled from cleavage at the ends of $\mathrm{Mu}$. Cell 51: 493-501.

Craigie, R., D.J. Arndt-Jovin, and K. Mizuuchi. 1985. A defined system for the DNA strand-transfer reaction at the initiation of bacteriophage $\mathrm{Mu}$ transposition: Protein and DNA sub- strate requirements. Proc. Natl. Acad. Sci. 82: 7570-7574.

Craigie, R., T. Fujiwara, and F. Bushman. 1990. The IN protein of Moloney murine leukemia virus processes the viral DNA ends and accomplishes their integration in vitro. Cell 62: 829-837.

Dixon, N.E. and A. Kornberg. 1984. Protein HU in the enzymatic replication of the chromosomal origin of Escherichia coli. Proc. Natl. Acad. Sci. 82: 424-428.

Doak, T.G., F.P. Doerder, C.L. Jahn, and G. Herrick. 1992. A proposed superfamily of transposase genes: Transposon-like elements in ciliated protozoa and a common "D35E" motif. Proc. Nat1. Acad. Sci. 91: 942-946.

Drelich, M., R. Wilhelm, and J. Mous. 1992. Identification of amino acid residues critical for endonuclease and integration activities of HIV-1 IN protein in vitro. Virology 188: 459468.

Engelman, A. and R. Craigie. 1992. Identification of conserved amino acid residues critical for human immunodeficiency virus type 1 integrase function in vitro. J. Virol. 66: 63616369.

Engelman, A., K. Mizuuchi, and R. Craigie. 1991. HIV-1 DNA integration: Mechanism of viral DNA cleavage and DNA strand transfer. Cell 67: 1211-1221.

Engelman, A., F.D. Bushman, and R. Craigie. 1993. Identification of discrete functional domains of HIV-1 integrase and their organization within an active multimeric complex. EMBO I. 12: 3269-3275.

Fayet, O., P. Ramond, P. Polard, M.F. Prere, and M. Chandler. 1990. Functional similarities between retroviruses and the IS3 family of bacterial insertion sequences? Mol. Microbiol. 4: $1771-1777$.

Fujiwara, T. and K. Mizuuchi. 1988. Retroviral DNA integration: Structure of an integration intermediate. Cell 54: 497504.

Gellert, M. and H. Nash. 1987. Communication between segments of DNA during site-specific recombination. Nature 325: 401-404.

Grindley, N.D.F. 1993. Analysis of a nucleoprotein complex: The synaptosome of $\gamma \delta$ resolvase. Science 262: 738-740.

Han Y.W., R.I. Gumport, and J.F. Gardner. 1993. Complementation of bacteriophage lambda integrase mutants: Evidence for an intersubunit active site. EMBO J. 12: 4577-4584.

Haniford, D.B., H.W. Benjamin, and N. Kleckner. 1991. Kinetic and structural analysis of a cleaved donor intermediate and a strand transfer intermediate in Tn10 transposition. Cell 64: 171-179.

Jones, K.S., J. Coleman, G.W. Merkel, T.M. Laue, and A.M. Skalka. 1992. Retroviral integrase functions as a multimer and can turn over catalytically. J. Biol. Chem. 267: 1603716040.

Kaufman, P.D. and D.C. Rio. 1992. P element transposition in vitro proceeds by a cut-and-paste mechanism and uses GTP as a cofactor. Cell 69: 27-39.

Kulkosky, J., K.S. Jones, R.A. Katz, J.P.G. Mack, and A.M. Skalka. 1992. Residues critical for retroviral integrative recombination in a region that is highly conserved among retroviral/retrotransposon integrases and bacterial insertion sequence transposases. Mol. Cell. Biol. 12: 2331-2338.

Lavoie, B.D., B.S. Chan, R.G. Allison, and G. Chaconas. 1991 Structural aspects of a higher order nucleoprotein complex: Induction of an altered DNA structure at the Mu-host junction of the Mu type 1 transpososome. EMBO J. 10: 30513059.

Leavitt, A.D., L. Shiue, and H.E. Varmus. 1993. Site-directed mutagenesis of HIV-1 integrase demonstrates differential effects on integrase functions in vitro. I. Biol. Chem. 268: 
2113-2119.

Lefemina, R.L., C.L. Schneider, H.L. Robbins, P.L. Callahan, K. Legrow, E. Roth, W.A. Schleif, and E.A. Emini. 1992. Requirement of active human immunodeficiency virus type 1 integrase enzyme for productive infection of human T-lymphoid cells. J. Virol. 66: 7414-7419.

Mizuuchi, K. 1992a. Polynucleotidyl transfer reaction in transpositional DNA recombination. I. Biol. Chem. 267: 2127321276.

1992b. Transpositional recombination: Mechanistic insights form studies of $\mathrm{Mu}$ and other elements. Annu. Rev. Biochem. 61: 1011-1051.

Mizuuchi, K. and K. Adzuma. 1991. Inversion of the phosphate chirality at the target site of Mu DNA strand transfer: Evidence for a one-step transesterification mechanism. Cell 66: 129-140.

Mizuuchi, M. and K. Mizuuchi. 1989. Efficient Mu transposition requires interaction of transposase with a DNA sequence at the $\mathrm{Mu}$ operator: Implications for regulation. Cell 58: $399-408$.

Mizuuchi, M., T.A. Baker, K. and Mizuuchi. 1991. DNase protection analysis of the stable synaptic complexes involved in Mu transposition. Proc. Natl. Acad. Sci. 88: 9031-9035.

1992. Assembly of the active form of the transposaseMu DNA complex: A critical control point in $\mathrm{Mu}$ transposition. Cell 70: 303-311.

Rådström, P., O. Skold, G. Swedberg, J. Flensburg, P.H. Roy, and L. Sundström. 1994. Transposon Tn5090 of Plasmid R751, which carries an integron, is related to $\mathrm{Tn} 7, \mathrm{Mu}$ and the retroelements. J. Bacteriol. 176: 3257-3268.

Robertson, H.M. 1993. The mariner transposable element is widespread in insects. Nature 362: 241-245.

Rowland, S.-J. and K.G. Dyke. 1990. Tn552, a novel transposable element from Staphylococcus aureus. Mol. Microbiol. 4: 961-975.

Surette, M.G. and G. Chaconas. 1989. A protein factor which reduces the negative supercoiling requirement in the $\mathrm{Mu}$ DNA strand transfer reaction is Escherichia coli integration host factor. I. Biol. Chem. 264: 3028-3034.

- 1991. Stimulation of the Mu DNA strand cleavage and intramolecular strand transfer reactions by the $\mathrm{Mu} B$ protein is independent of stable binding of the $\mathrm{Mu} \mathrm{B}$ protein to DNA. J. Biol. Chem. 266: 17306-17313.

. 1992. The Mu transpositional enhancer can function in trans: Requirement of the enhancer for synapsis but not strand cleavage. Cell 68: 1101-1108.

Surette, M.G., S.J. Buch, and G. Chaconas. 1987. Transpososomes: Stable protein-DNA complexes involved in the in vitro transposition of bacteriophage Mu DNA. Cell 49: 253262.

Surette, M.G., T. Harkness and G. Chaconas. 1991. Stimulation of the $\mathrm{Mu}$ A protein-mediated strand cleavage reaction by the $\mathrm{Mu} \mathrm{B}$ protein, and the requirement of DNA nicking for stable type 1 transpososome formation. In vitro transposition characteristics of mini-Mu plasmids carrying terminal base pair mutations. J. Biol. Chem. 266: 3118-3124.

van Gent, D.C., A.M. Antoinette, O. Groeneger, and R.H.A. Plasterk. 1992. Mutational analysis of the integrase protein of human immunodeficieny virus type 2. Proc. Natl. Acad. Sci. 89: 9598-9602.

van Gent, D.C., C. Vink, A.A.M. Oude Groeneger, and R.H.A. Plasterk. 1993. Complementation between HIV integrase proteins mutated in different domains. EMBO J. 12: 32613267.

Vink, C., E. Yeheskiely, G.A. van der Marel, J.H. van Boom, and R.H. Plasterk. 1991. Site-specific hydrolysis and alcoholysis of human immunodeficiency virus DNA termini mediated by the viral integrase protein. Nucleic Acids Res. 19: 66916698 . 


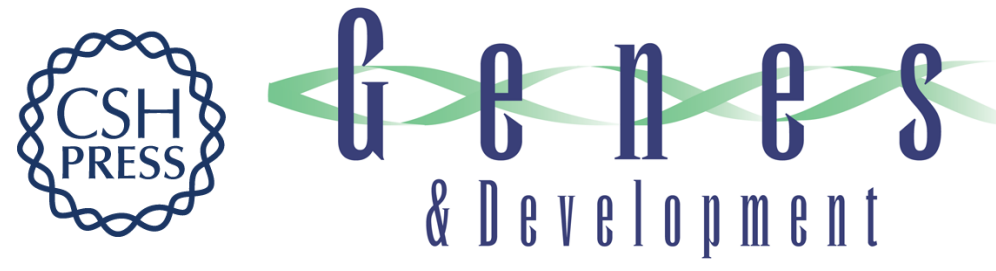

\section{Complete transposition requires four active monomers in the mu transposase tetramer.}

T A Baker, E Kremenstova and L Luo

Genes Dev. 1994, 8:

Access the most recent version at doi:10.1101/gad.8.20.2416

References This article cites 52 articles, 21 of which can be accessed free at:

http://genesdev.cshlp.org/content/8/20/2416.full.html\#ref-list-1

License

Email Alerting

Service

Receive free email alerts when new articles cite this article - sign up in the box at the top right corner of the article or click here.

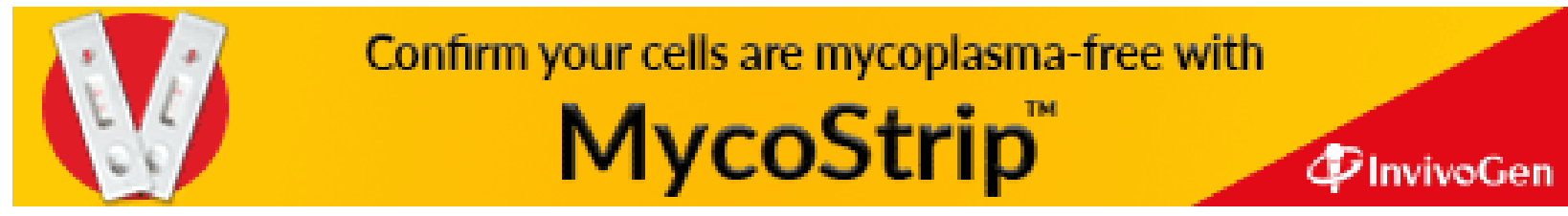

